\title{
Independence of genetic subdivison and variation for coil in Partula suturalis
}

\author{
M. S. Johnson*, \\ J. Murray† and \\ B. Clarke
}

* Department of Zoology, University of Western
Australia, Nedlands, Western Australia 6009 ,
Australia.
+ Department of Biology, University of Virginia,
Charlottesville, Virginia 22901 , U.S.A.
$\ddagger$ Department of Genetics, University of Nottingham,
Nottingham NG7 2RD U.K.

In Partula suturalis, populations monomorphic for dextral shells are separated by steep clines from those monomorphic for sinistral shells. Because the difference in chirality is genetically simple, and because it acts to promote reproductive isolation, there is special interest in determining whether the "area effects" for coil are associated with other genetic differences. We examined geographic variation of allozymes at 23 loci throughout the range of $P$. suturalis. Neither the pattern nor the amount of allozyme variation are related to the area effects for coil. There is little evidence of allozymic differences between shells of different coils within polymorphic populations. Although chirality may be important in premating isolation, it appears to be ineffective in promoting genetic divergence.

\section{INTRODUCTION}

The land snail Partula suturalis is polymorphic for the direction of shell coiling. The polymorphism is due to a single locus, with the allele for sinistrality dominant, but with the added complication that each snail's phenotype is determined by its mother's genotype (Murray and Clarke, 1976). $P$. suturalis is endemic to the island of Moorea, in French Polynesia, where the distribution of the sinistral and dextral morphs shows a striking pattern. Across the north of the island, populations are monomorphic for sinistral shells, whereas in the south they are monomorphic for dextral shells. These "area effects" are connected by relatively narrow zones containing polymorphic populations (Murray and Clarke 1980).

The direction of shell coiling affects the mating behaviour of the snails, so that individuals of opposite coils are less likely to mate than are those with the same coil (Lipton and Murray, 1979; Johnson, 1982). Therefore, snails with the less common coil in a polymorphic population are less likely to mate, and they produce fewer young (Johnson, 1982). This positive frequency dependent selection explains the monomorphism of most populations, and the narrowness of the clines connecting the dextral and sinistral populations.

The polymorphism is of special interest, because it represents a genetically simple premat- ing isolating mechanism. Given the interest in behavioural isolation as an initiator of speciation (Bush, 1975; Tauber and Tauber, 1977), it is important to determine whether the sinistral and dextral groups of populations are in the process of speciating. In this context, we wish to know whether the polymorphism for chirality is independent of, or associated with, other types of genetic differentiation, either geographically or within polymorphic populations. We have therefore examined allozyme polymorphisms within and between the areas of sinistrality and dextrality. As well as estimating the degree of genetic differentiation associated with variation in coil, the study provides a general survey of genetic subdivision in $P$. suturalis.

\section{MATERIALS AND METHODS}

A group of 23 samples, drawn from the entire distribution of $P$. suturalis, was used to examine broad patterns of geographic variation. These samples, each consisting of approximately 20 snails, have already been used in comparisons among species of Partula on Moorea (Johnson et al., 1986). We obtained an additional 22 samples from the transition zone between the sinistral and dextral areas, in order to examine in detail any genetic changes at the boundary. The average size 
of these later samples was about 50 , allowing the comparison of allelic frequencies in sinistral and dextral individuals. All the sampling sites are shown in fig. 1.

Genetic variation in the enzymes was examined electrophoretically, as described previously (Johnson et al., 1977, 1986). In the broad geographic survey, 23 loci were scored. In the transition zone, 9 polymorphic loci were chosen for study: Got-1, Idh-1, Ldh, Mpi, Np, Pep-6, Pgi, Pgm-1, Pgm-2. Because of the large number of populations, complete tables of allelic frequencies are not presented. They are available from any of the authors, and copies have been deposited in the library of the Royal Society, London.

For each allele at each locus, allelic frequencies in monomorphic sinistral and monomorphic dextral populations were compared, using MannWhitney U-tests. The area of sinistral populations is complex, and includes three disjunct sets of populations in the northwest, north-central, and northeast parts of the island. Consequently, the U-tests were made at two levels, using either the entire set of sinistral populations or only those from the northwest area. Greater density of sampling was conducted in the northwest, to avoid possible confusion due to localised hybridisation with other species in the northeast (Murray and Clarke, 1980). Product-moment correlations of allozymic frequencies with the proportion of dextral individuals were calculated for populations in the transition zone.

Allozymic differentiation among the 23 populations was measured using Nei's unbiased genetic identity, $I$ (Nei, 1978). The overall pattern of geographical variation was summarised by clustering, using the unweighted pair-group method with arithmetic averages, UPGMA (Sneath and Sokal, 1973). Although this method provides a useful summary of the levels of similarity, it can produce apparently distinct clusters, even when variation is continuous; and it often represents close relationships better than higher-order groupings. To provide an alternative summary of geographical variation we performed a principal coordinates analysis of the allozymic frequencies, using the



Figure 1 Map of Moorea, showing sampling sites for $P$. suturalis. Samples with double-digit codes were included in the full survey of 23 loci; those with three-digit codes were examined for 9 loci. Open symbols indicate sinistral populations; solid symbols, dextral populations; and half-solid, polymorphic populations. 
GENSTAT routine at the Western Australian Regional Computing Centre. This analysis was based on a matrix of Manhattan distances, derived from range-coded allelic frequencies at the 23 loci (see Sneath and Sokal, 1973).

The average values of $I$ were determined in order to make comparisons between populations of the same or of opposite coil. Such comparisons were complicated by the fact that variation in $I$ is already known to be related to geographical distance between populations of $P$. suturalis (Johnson et al., 1986). Consequently, we used an analysis of covariance (Sokal and Rohlf, 1981) to compare the average value of $I$ within and between areas.

We also compared the proportions of allozymic alleles in dextral and sinistral snails taken from the same polymorphic population, using Chisquare tests of contingency tables.

\section{RESULTS}

Geographical variation at each of the polymorphic loci is shown in fig. 2 . The most extreme divergence is found at the Pgm-2 locus. The Pgm-2 $2^{0.87}$ allele is nearly fixed in the extreme southern populations, and absent elsewhere. The two southernmost populations, on the slopes of Mount Ahutau, diverge also at other loci. For example, they are the only populations lacking the $P g m-1^{0.95}$ allele. These differences occur within the dextral area, not between the dextral and sinistral groups. There are no striking differences between these two groups, but there are some statistically significant $(P<0.05)$ associations. The most obvious is the generally lower frequency of the $P g i^{0.50}$ allele in the dextral populations. Another is the high frequency of $N p^{1.00}$ in the northwestern group of sinistrals. A few associations are marginal: Got$1^{1 \cdot 50}$ and $P e p-6^{1 \cdot 00}$ are generally at higher frequencies in the sinistral populations, but there is much overlap; $I d h^{1 \cdot 16}$ is marginally more common in the dextral area, but also with much overlap; $M p i^{1 \cdot 07}$ has frequencies above 0.02 only in dextral populations, but half of these populations lack the allele.

Within the transition zone, allelic frequencies at three loci are significantly correlated with the proportion of dextral shells (fig. 3). The frequency of Got-1 ${ }^{1.50}(r=-0.57)$ and Pep- $6^{1.00}(r=-0.40)$ are negatively correlated with the frequency of dextrals, while $P g i^{0.50}(r=0.45)$ is positively correlated. None of these correlations is strong, but they are consistent with the broader geographical association with the coil area effects, and they confirm that the three loci are changing in the region of change in coil.
Based on the full set of 23 loci, the genetic identities show geographic patterns, but they are not associated with the variation in chirality (fig. 4). The major geographical difference is between the populations from Mount Ahutau and all the rest, both dextral and sinistral. Each of the other major clusters includes both sinistral and dextral populations. The only group relevant with respect to the coil area effect is that which includes all the northwestern dextral populations, the transition zone, and the western-most dextral population, indicating that there is divergence along the same axis as the northwest-southeast transition between dextral and sinistral areas.

The continuity of geographic variation and its independence of the variation in chirality are shown clearly by the principal coordinates analy. sis, in which we do not find distinct clusters of populations. The first two axes of variation, representing 22.5 per cent and 15.9 per cent of the total variation, show clinal patterns across the island (fig. 5). The first axis shows a general change roughly in a NNE-SSW direction. The second axis represents variation from the center of the island outwards. Clinal variation from the southeast to the northwest is evident on each of these axes, but is not associated with the change in coil area. The third and fourth axes $(12.4$ per cent and 10.3 per cent of the variation) do not show areal groupings, and the remaining axes each represent less than 7 per cent of the variation.

In a general way, allozymic similarity decreases with increasing distance between populations. It does so both within and between coil areas $\left(r^{2}>\right.$ 0.79 in each case; fig. 6). Neither the slope nor the adjusted $Y$ values differ between the two sets of comparisons. In order to avoid inflating the estimates of divergence within the dextral area, comparisons involving the peculiar populations from Mount Ahutau have been excluded from this analysis. It is clear that there is no decrease in overall allozymic similarity associated with the transition between dextral and sinistral populations, and the patterns of geographic variation of the allozymes transcend the variation in chirality.

In the transition zone there were only two cases where allelic frequencies differed between the sinistrals and dextrals from the same population. Both occurred in sample 546 (table 1): in the dextral snails the frequency of $N p^{1 \cdot 00}$ was higher and the frequency of $P e p-6^{1 \cdot 00}$ was lower than they were in the sinistrals. These associations are due to the presence of 3 sinistral individuals that were homozygous for both $N p^{0.84}$ and $P e p-6^{0.85}$ 

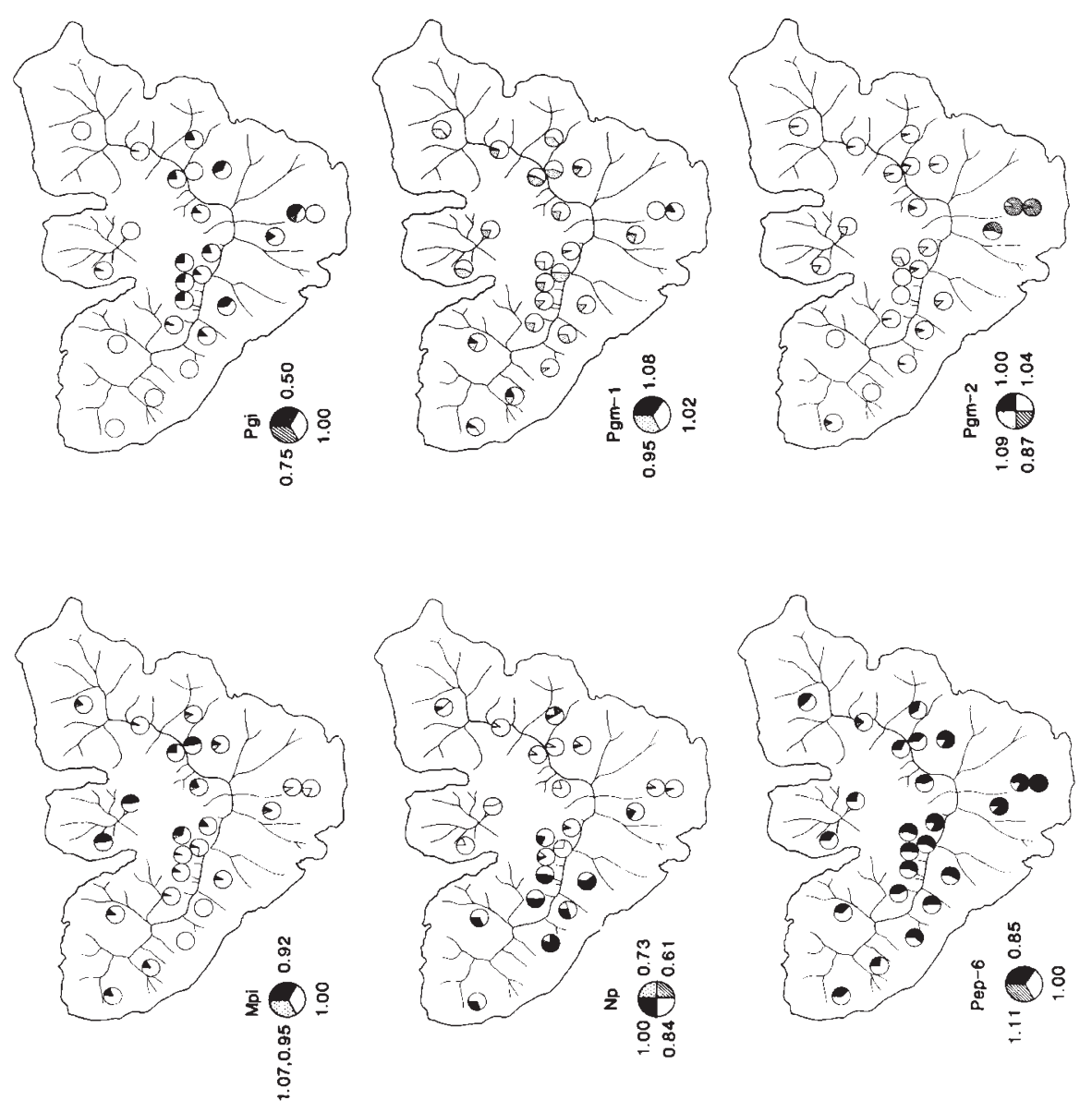

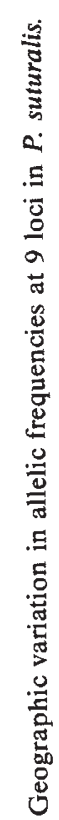
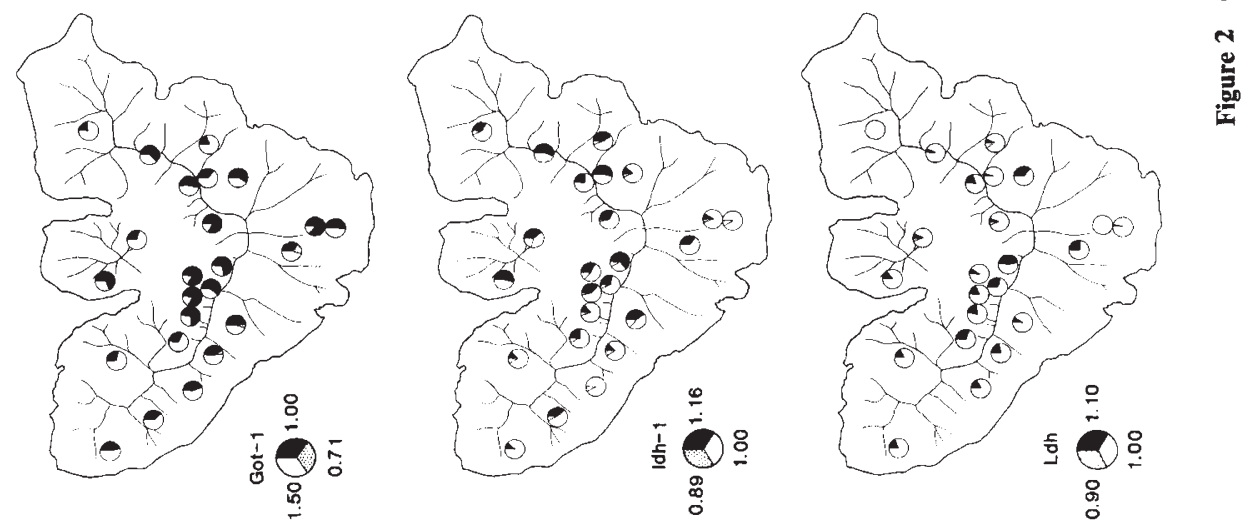




Figure 3 Correlations of allelic frequencies at 3 loci with the proportion of dextral $P$. suturalis in the transition zone between dextral and sinistral areas.

\section{DISCUSSION}

Although there are patterns to the geographic variation of allozymes in $P$. suturalis, they do not seem to be related to the variation in chirality. The greatest divergence is shown by the populations from Mount Ahutau, within the area of dextral populations. Even if we ignore these extreme populations, the major geographic variation in allozymes is not between dextral and sinistral areas. Variation at three loci is correlated with variation in the proportion of dextral individuals, both between areas and in the transition zone; but those associations are part of a broader pattern of variation along a northwest-southeast axis, rather than a pervasive genetic difference between coil areas. Similarly, shell shape, which varies pleiotropically with coil, is geographically independent of it (Johnson, 1987). The independence of variation in chirality from other genetic variation is important in understanding the origin of the "area effect" and the significance of the change in chirality for speciation.

The geographic distribution of dextrality in $P$. suturalis follows closely the distribution of sinistral species belonging to the $P$. suturalis complex, and a reasonable interpretation is that there has been selection for premating isolation. Because of its role in courtship, the direction of coil is subject to positive frequency dependent selection, resulting in monomorphism of most populations (Johnson, 1982). In the light of this disadvantage of the rarer coil type, there are several plausible explanations for the origin of the populations that differ in coil, including large-scale geographic isolation, divergence in small isolates, and parapatric divergence. It is always difficult to determine the history of clinal variation, since both secondary contact following geographical isolation and primary contact resulting from parapatric divergence produce the same result (Endler, 1977; Barton and Hewitt, 1985). Nevertheless, secondary contact is typically accompanied by several concordant clines in different traits (Barton and Hewitt, 1985). Geographical associations among allozyme loci, or between allozymes and shell characters, have been taken as evidence for past geographic isolation among populations of Cepaea nemoralis (Johnson, 1976; Ochman et al., 1983). Accepting the absence of concordant variation in other traits, we can argue that the coil area effect in $P$. suturalis is unlikely to have originated through large-scale geographic separation. Parapatric divergence would produce parallel variation in different traits only if the traits were subject to selection associated with the same environmental gradients, or if there were epistatic interactions with loci so associated.

Table 1 Allelic frequencies at the $N p$ and $P e p-6$ loci in dextral and sinistral individuals within sample 546 of $P$. suturalis. $N p^{0.73}$ was excluded from the $\chi^{2}$ test, because of its low frequency

\begin{tabular}{lllllll}
\hline & Sample size & $N p^{1.00}$ & $N p^{0.84}$ & $N p^{0.73}$ & \multicolumn{1}{c}{$P e p \cdot 6^{1 \cdot 00}$} \\
\hline $\begin{array}{lllll}\text { Dextrals } \\
\text { Sinistrals }\end{array}$ & 53 & 0.67 & 0.30 & 0.03 & 0.29 & 0.71 \\
$\chi^{2}$ & 17 & 0.47 & 0.50 & 0.03 & 0.53 & 0.47 \\
\hline
\end{tabular}

$* P<0.05 ; * * P<0.01$. 


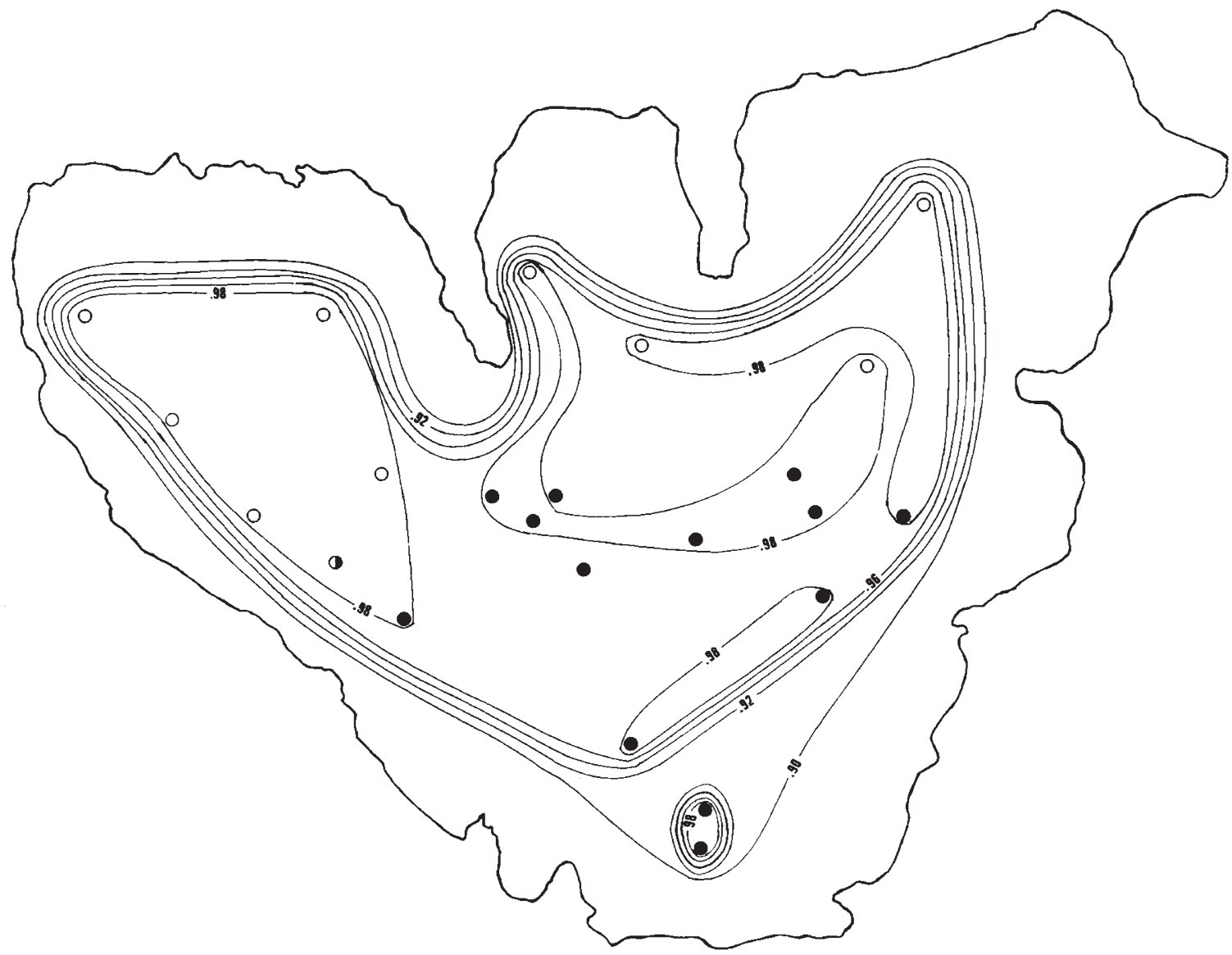

Figure 4 Isopleths of average genetic identity (UPGMA clustering) among populations of $P$. suturalis. Open symbols indicate sinistral populations; solid symbols, dextral populations; and half-solid, polymorphic populations.

Neither of these possibilities is likely for geographical variation in coil, which has an unusual selective basis. Consequently, the allozyme data do not tell us whether the divergence in chirality occurred parapatrically or through spreading from a small isolate. Neither of these possibilities is likely to entail associated allozymic change.

Whatever the geographical history of the change in chirality, it is unlikely that the coil area effect represents incipient speciation. Increased allozymic divergence is not associated with the change of coil, indicating that such a change poses little, if any, barrier to gene flow. Given the limited vagility of these snails, this observation is not surprising. Even within populations there is little evidence for subdivision based on the isolating effects of differences of coil. The linkage disequilibrium found in one sample is due to 3 peculiar individuals, and could be due to spatial subdivision on a fine scale, or even self-fertilisation. In any case it is exceptional, indicating that subdivision associated with coil is not the rule. If behavioral isolation is to have significant effects on gene flow, the isolation must be very strong (Barton and Hewitt, 1985). The isolation caused by change of coil is weakened by the delayed inheritance. The resulting disconnection between genotype and phenotype means that even complete assortative mating by coil would not prevent gene flow between sinistrals and dextrals. Thus, while chirality may be significant where there is postmating isolation, it is unlikely to initiate divergence. The absence of concordant differentiation in other traits supports the view that the low fecundity crosses between coils is due to the low frequency of mating (Johnson, 1982), rather than to other genetic differences between sinistral and dextral subpopulations.

Although they put the coil "area effect" in a clearer context, the geographical relationships of allozymes in $P$. suturalis pose some difficult questions. The large-scale patterns are particularly sur- 

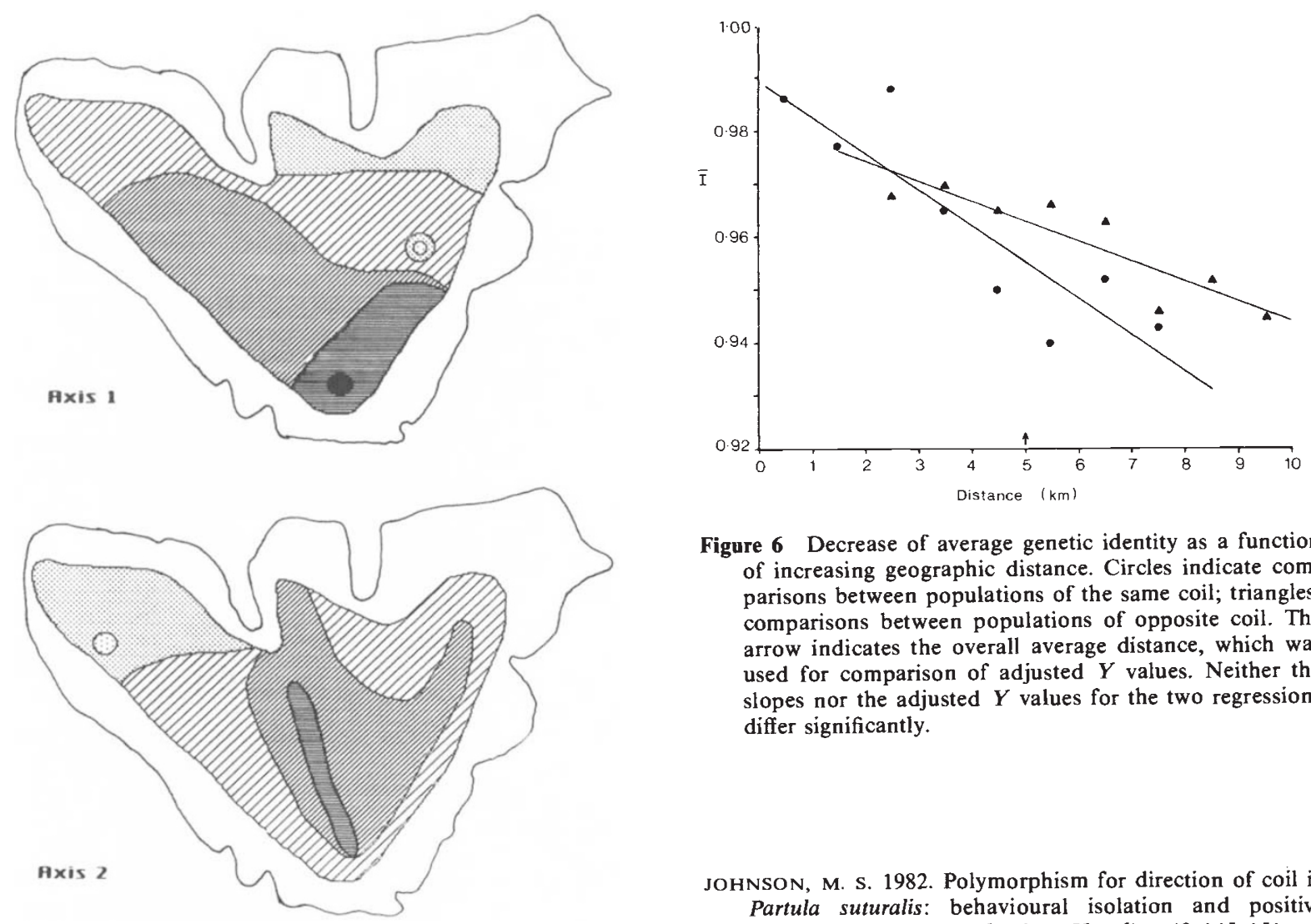

Figure 6 Decrease of average genetic identity as a function of increasing geographic distance. Circles indicate comparisons between populations of the same coil; triangles, comparisons between populations of opposite coil. The arrow indicates the overall average distance, which was used for comparison of adjusted $Y$ values. Neither the slopes nor the adjusted $Y$ values for the two regressions differ significantly.

Figure 5 Isopleths of scores on the first 2 principal coordinate axes. Scores are indicated by the type of shading: less than -2 , solid; -2 to -1 , dense horizontal; -1 to 0 , moderate diagonal; 0 to +1 , sparse diagonal; +1 to +2 , dense dots; greater than +2 , sparse dots.

prising, given the low vagility of land snails in general and Partula, in particular (Murray and Clarke, 1984). This problem is under investigation.

Acknowledgements This work was funded by the Australian Research Grants Scheme, the U.S.-Australian Cooperative Science Program (NSF 2AS-30), and the Science and Engineering Research Council. Bill Barendse provided comments on the manuscript.

\section{REFERENCES}

BARTON, N. H., AND HEWITT, G. M. 1985. Analysis of hybrid zones. Ann. Rev. Ecol. Syst., 16, 113-148.

BUSH. O. L. 1975. Modes of animal speciation. Ann. Rev. Ecol. Syst., 6, 339-364.

ENDLER, J. A. 1977. Geographic Variation, Speciation, and Clines, Princeton Univ. Press.

JOHNSON, M. S. 1976. Allozymes and area effects in Cepaea nemoralis on the Berkshire downs. Heredity, 36, 105-121.

JOHNSON, M. S. 1982. Polymorphism for direction of coil in Partula suturalis: behavioural isolation and positive frequency dependent selection. Heredity, 49, 145-151.

JOHNSON, M. S. 1987. Adaptation and rules of form: chirality and shape in Partula suturalis. Evolution, in press.

JOHNSON, M. S., CLARKE, B., AND MURRAY, J. 1977. Genetic variation and reproductive isolation in partula. Evolution, 31, 116-126.

JOHNSON, M. S., MURRAY, J., AND ClARKE, B. 1986. Allozymic similarities among species of Partula on Moorea. Heredity, 56, 319-327.

LIPTON, C. S., AND MURRAY, J. 1979. Courtship of land snails of the genus Partula Malacologia, 19, 129-146.

MURRAY, J., AND CLARKE, B. 1976. Supergenes in polymorphic land snails. II. Partula suturalis. Heredity, 37, 271-282.

MURRAY, J., AND ClARKE, B. 1980. The genus Partula on Moorea: speciation in progress. Proc. Roy. Soc. Lond. B, 211, 83-117.

MURRAY, J., AND CLARKE, B. 1984. Movement and gene flow in Partula taeniata. Malacologia, 25, 343-348.

NEI, M. 1978. Estimation of average heterozygosity and genetic distance from a small number of individuals. Genetics, 89, 583-590.

OCHMAN, H., JONES, J. S., AND SELANDER, R. K. 1983. Molecular area effects in Cepaea. Proc. Nat. Acad. Sci. U.S.A., 80, 4189-4193.

SNEATH, P. H. A., AND SOKAL, R. 1973, Numerical Taxonomy, Freeman, San Francisco.

SOKAL, R. R., AND ROHLF, F. J. 1981. Biometry, 2nd ed. Freeman, San Francisco.

TAuber, C. A., AND TAuber, M. J. 1977. A genetic model for sympatric speciation through habitat diversification and seasonal isolation. Nature, 268, 702-705. 